\title{
How to Develop Psychiatry Investigation into a World Class Journal
}

\author{
Heon-Jeong Lee, ${ }^{1,2}$ Editor-in-Chief \\ ${ }^{1}$ Department of Psychiatry, Korea University College of Medicine, Seoul, Republic of Korea \\ ${ }^{2}$ Chronobiology Institute, Korea University, Seoul, Republic of Korea
}

This is my last editorial as the Editor-in-Chief of Psychiatry Investigation. It has been a pleasure and honor that important milestones such as indexing by the Science Citation IndexExpanded in April 2009, the Pubmed Central in December 2009, and the Social Science Citation Index in April 2012, were achieved during my working years. ${ }^{1}$ Psychiatry Investigation has been successful thanks to the outstanding performances of the previous Editors-in-Chief: Byoung-Hoon Oh (2004-2005), Chan-Hyung Kim (2006-2009), Jun-Soo Kwon (2009-2011), Chang-Uk Lee (2012-2015), and Dai-Jin Kim (2016-2017). After I took the position of Editor-in-Chief of the journal two years ago, we began to publish the journal every month as an important step toward becoming a world class journal.

However, much effort would be needed in future to achieve the aim of developing it into a leading journal. First, it is necessary to ensure regional diversity by having authors from across the world. According to the national distribution of published papers in Psychiatry Investigation in the last two years, authors from a total of 28 countries published their papers, and 73.3 percent of them were from South Korea, which implies that the articles are largely contributed by authors concentrated in the same area in terms of regional diversity. This compares with 57.1 percent of articles in "Psychiatry and Clinical Neurosciences" in Japan during the same period, which also indicates that there is a higher ratio of domestic authors in Psychiatry Investigation. ${ }^{2}$ However, according to the national distribution of paper submissions for Psychiatry Investigation, the number of submissions from foreign countries accounts for one-third of the total submissions. This implies that high quality papers that can pass the review process and get published must be submitted from around the world.

It is imperative to find a solution to address this problem. One way could be to increase the invitation of papers from

(c) This is an Open Access article distributed under the terms of the Creative Commons Attribution Non-Commercial License (https://creativecommons.org/licenses/by$\mathrm{nc} / 4.0$ ) which permits unrestricted non-commercial use, distribution, and reproduction in any medium, provided the original work is properly cited. influential researchers. As the number of new journals has increased exponentially in recent years, readers have difficulty in reading and updating their knowledge with the large number of papers in the field of psychiatry, other than those that they are specifically interested in. Therefore, it is a good way to increase journal impact factor by inviting experts from various fields to present a review paper to meet the reader's interest and bring about an increase in citations. It is true that the JCR impact factor of the journal has shown a slight decrease because the volume of publications has increased in recent years. We have recently made an effort to increase the impact factor again through various strategies. As a result of these efforts, it is expected to be around 1.6 in the JCR impact factor for 2019, which is scheduled to be announced in June 2020 .

The way forward for a latecomer like Psychiatry Investigation, to grow into a world class journal, is to publish content that conforms to the new directions of psychiatry. The scope of Psychiatry Investigation is wide and encompasses the entire field. Taking an interest in new areas that are future-oriented will lead to newer opportunities. It is advisable that we increase the quota for areas that will lead to new diagnosis and treatment and health promotion opportunities, such as digital therapeutics, internet of medical things, and artificial intelligence. $^{3-6}$

I am sure that if we respond positively to the development of psychiatry in future, our journal, Psychiatry Investigation, will continue to develop and establish itself as one of the leading psychiatry journals in Asia.

\section{ORCID iD}

Heon-Jeong Lee https://orcid.org/0000-0002-9560-2383

\section{REFERENCES}

1. Lee HJ. Psychiatry Investigation: 15 years of challenges. Psychiatry Investig 2018;15:1-2.

2. Web of Science citation report for Psychiatry Investigation and Psychiatry and Clinical Neurosciences. Available at: http://apps.webofknowledge.com. Accessed December 1, 2019.

3. Cho $\mathrm{CH}$, Lee $\mathrm{HJ}$. Could digital therapeutics be a game changer in psy- 
chiatry? Psychiatry Investig 2019;16:97-98.

4. Ryu S, Lee H, Lee DK, Park K. Use of a machine learning algorithm to predict individuals with suicide ideation in the general population. Psychiatry Investig 2018;15:1030-1036.

5. Lee HA, Lee HJ, Moon JH, Lee T, Kim MG, In H, Cho CH, et al. Com- parison of wearable activity tracker with actigraphy for sleep evaluation and circadian rest-activity rhythm measurement in healthy young adults. Psychiatry Investig 2017;14:179-185.

6. Lee HJ. Chronobiology, the future of medicine. Chronobiol Med 2019;1:1-2. 